\title{
A remark on some fuchsian groups
}

\author{
Murat Beşenk \\ Pamukkale University, Faculty of Arts and Sciences, Department of Mathematics, Denizli, Turkey
}

Received: 30 November 2017, Accepted: 13 December 2017

Published online: 30 June 2018.

\begin{abstract}
In this paper we study combinatorial structures of some Fuchsian groups. We examine fundamental domains, group actions and genus for these groups.
\end{abstract}

Keywords: Group action, cusp, fundamental domain, genus.

\section{Introduction}

In [1-5] authors investigate some important classes of modular group subgroups of finite index. It is known that given a group action, representation gives further means to study the object being acted upon, yielding more information about these groups. Thus group representations are an organizing principle in the theory of finite groups [6-7]. The studies will also be of help to various fields. To combinatorics, calculation of cycle index formulas will enhance processes by which we organize sets so that we can interpret and apply the data they contain. And also these groups use very often in modular forms [10].

Now we consider hyperbolic plane model. The complex upper half plane is the set $\mathbb{H}$ of complex numbers with positive imaginary part $\mathbb{H}:=\{\tau \in \mathbb{C}: \operatorname{Im}(\tau)>0\}$. As E. Beltrami and $\mathrm{H}$. Poincare noticed, $\mathbb{H}$ can present as a model for non-Euclidean hyperbolic plane geometry. In the group theory a point $s \in \mathbb{R} \cup\{\infty\}$ is known cusp for Möbius transformations if it is the fixed point of a parabolic element. The extended upper half-plane is the union of $\mathbb{H}$ with the set of cusps $\mathbb{Q} \cup\{\infty\}$, and we refer to is as $\mathbb{H}^{*}$. We observe that $\mathbb{H}$ admits a natural structure of Riemann surface. Actually, it is one of the only three simply connected Riemann surface, up to biholomorphic isomorphism, though resides in the structure given by the action of certain multiplicative groups of matrices on these domains. Several groups of matrices will appear throughout this work.

Definition 1. The general linear group $G L(2, \mathbb{C})$ acts on $\mathbb{P}^{1}=\mathbb{C} \cup\{\infty\}$ by linear fractional transformations in the following way:

$$
G L(2, \mathbb{C}) \times \mathbb{P}^{1} \longrightarrow \mathbb{P}^{1},(\gamma, z) \rightarrow \gamma(z)=\frac{a z+b}{c z+d} \text { where } \gamma=\left(\begin{array}{ll}
a & b \\
c & d
\end{array}\right)
$$

In the preceding definition, we adopt the convention that $\lim _{z \rightarrow \infty} \frac{a z+b}{c z+d}=\frac{a}{c}$ and $\frac{w}{0}=\infty$ for all $w \in \mathbb{P}^{1}$. We want to obtain an action on $\mathbb{H}$. In this case, we restrict the coefficients of the real matrices to real numbers. 
Let $\operatorname{PSL}(2, \mathbb{R})$ denote the group consisting of all linear fractional transformations

$$
\Pi: \tau \rightarrow \frac{a \tau+b}{c \tau+d} \text { where } a, b, c, d \text { are real numbers and } a d-b c=1 .
$$

In terms of matrix representation, the elements of $\operatorname{PSL}(2, \mathbb{R})$ correspond to the matrices

$$
\pm\left(\begin{array}{ll}
a & b \\
c & d
\end{array}\right) ; a, b, c, d \text { are real numbers and } a d-b c=1 .
$$

Any function from $\mathbb{H}$ to itself which preserves distance is known an isometry. And also the group of all isometries of $\mathbb{H}$ is named by $\operatorname{Isom}(\mathbb{H})$. Moreover this group is generated by $\operatorname{PSL}(2, \mathbb{R})$ together with the map $z \rightarrow-\bar{z}$ and therefore we have the index $|\operatorname{Isom}(\mathbb{H}): \operatorname{PSL}(2, \mathbb{R})|=2$.

\section{Discrete subgroups}

First of all we know from the literature discrete subgroups of $S L(2, \mathbb{R})$ abound, but those of interest to number theorists are rather special [8-9]. Discrete groups play a important role especially in dynamical systems where they arise as transformation groups in a rich variety of instances: geometry, ergodic theory, elliptic integral theory, cryptology etc. Recently discrete groups have been used in various applied areas. Consequently in more detail, the theory of discrete subgroups in case continuous groups includes such applications as arithmetic and algebraic groups, fundamental groups and covering spaces, Teichmuller spaces, Fuchsian groups, Kleinian groups, non-Euclidean geometry, automorphic forms, modular and cusp forms, Hecke operators, moduli spaces, deformation spaces, the theory of boundary spaces for random walks on infinite graphs, suborbital graphs and more.

Definition 2. A subgroup $G$ of $P S L(2, \mathbb{C})$ is said to be discrete if it contains no sequence of matrices converging element wise to the identity. Discrete subgroup of $P S L(2, \mathbb{R})$ are called Fuchsian groups.

Definition 3. The group $S L(2, \mathbb{Z})$ is called the full modular group.

The modular group $\Gamma=P S L(2, \mathbb{Z})=S L(2, \mathbb{Z}) /\{ \pm I\}$, is the subgroup of $\operatorname{PSL}(2, \mathbb{R})$ with integral coefficients. Clearly we can say that $\operatorname{PSL}(2, \mathbb{Z})$ is a Fuchsian group. It is generated by the matrices

$$
S=\left(\begin{array}{cc}
0 & -1 \\
1 & 0
\end{array}\right) ; \quad T=\left(\begin{array}{cc}
0 & -1 \\
1 & 1
\end{array}\right)
$$

with defining relationships $S^{2}=T^{3}=-I$, where $I$ is the identity matrix. This is the automorphism group of the upper half plane $\mathbb{H}$. Notice that if $g=\left(\begin{array}{ll}a & b \\ c & d\end{array}\right) \in \Gamma$ and $\tau \in \mathbb{H}$, then $g . \tau=\left(\begin{array}{ll}a & b \\ c & d\end{array}\right) \tau=\frac{a \tau+b}{c \tau+d}$. Hence $\Gamma$ acts on the $\mathbb{H}$. Indeed $\operatorname{Im}(g . \tau)=\frac{1}{|c \tau+d|^{2}} \operatorname{Im} \tau>0$ is obtained. That is, every $g \in \Gamma$ preserves $\mathbb{H}$. $S L(2, \mathbb{Z})$ is obviously a discrete subgroup of $S L(2, \mathbb{R})$, and so are all its subgroups as a consequence. And also $S L(2, \mathbb{Z})$ acts on $\mathbb{H}^{*}$.

Similarly the group $\operatorname{PSL}(2, \mathbb{C})$ acts on 3-dimensional hyperbolic space $\mathbb{H}^{3}=\{z+t j: z=a+i b \in \mathbb{C}, t>0\}$, that is, $\mathbb{H}^{3}=\mathbb{C} \times \mathbb{R}^{+}$. Again we have the index $\left|\operatorname{Isom}\left(\mathbb{H}^{3}\right): \operatorname{PSL}(2, \mathbb{C})\right|=2$. 
Remark. One can explain that this is set of relations, and it can be easily seen that $\Gamma /\{ \pm 1\}$ is the free product of the cyclic group of order 2 generated by $S$ and the cyclic group of order 3 generated by $S T$.

Definition 4. For any positive integer $N$, we define

$$
\Gamma(N)=\left\{\left(\begin{array}{ll}
a & b \\
c & d
\end{array}\right) \in S L(2, \mathbb{Z}):\left(\begin{array}{ll}
a & b \\
c & d
\end{array}\right) \equiv\left(\begin{array}{ll}
1 & 0 \\
0 & 1
\end{array}\right)(\bmod N)\right\}
$$

and call it the principal congruence subgroup of level $N$.

A congruence subgroup of $S L(2, \mathbb{Z})$ is a subgroup $\Gamma$ containing $\Gamma(N)$ for some $N$ : the minimum such $N$ is called the level of $\Gamma$. For example the most important families of congruence subgroups

$\Gamma_{0}(N)=\left\{\left(\begin{array}{ll}a & b \\ c & d\end{array}\right) \in S L(2, \mathbb{Z}):\left(\begin{array}{ll}a & b \\ c & d\end{array}\right) \equiv\left(\begin{array}{l}* * \\ 0\end{array}\right)(\bmod N)\right\}, \Gamma_{1}(N)=\left\{\left(\begin{array}{ll}a & b \\ c & d\end{array}\right) \in S L(2, \mathbb{Z}):\left(\begin{array}{ll}a & b \\ c & d\end{array}\right) \equiv\left(\begin{array}{ll}1 & * \\ 0 & 1\end{array}\right)(\bmod N)\right\}$

for any positive integer $N$, which is the level of both subgroups. We can easily seen that $\Gamma(1)=\Gamma_{0}(1)=\Gamma_{1}(1)=S L(2, \mathbb{Z})$ is the only congruence subgroup of level 1 . Indeed, because $S L(2, \mathbb{Z} / 1 \mathbb{Z})$ is trivial group, $\Gamma(1)=\operatorname{ker}(\mathbb{Z}) \rightarrow S L(2, \mathbb{Z} / 1 \mathbb{Z})$ must be all of $S L(2, \mathbb{Z})$. As $S L(2, \mathbb{Z})=\Gamma(1) \subset \Gamma_{1}(1) \subset \Gamma_{0}(1) \subset S L(2, \mathbb{Z})$, we must have this equality.

We know that every abelian Fuchsian group is cyclic. Therefore all non identity elements of $G$ have the same fixed point set and are of same type: parabolic, elliptic or hyperbolic. The congruence subgroups have been studied extensively, especially, by Felix Klein, Robert Fricke and many others. These groups are basic to the theory of the elliptic modular functions.

\section{Fundamental domains}

It is known that a discontinuous group is discrete. $\Gamma$ acts properly discontinuously on $\mathbb{H}$, that is, for any two distinct points $x, y \in \mathbb{H}$, there exist open neighbourhoods $U, V$ containing $x, y$ respectively such that the number of group elements $g \in \Gamma$ with $g U \cap V \neq \varnothing$ is finite. For such an action there is a notion of fundamental domain: a subset $F$ of $\mathbb{H}$ such that

(i) $\mathbb{H}=\bigcup \gamma F$, for all $\gamma \in \Gamma$,

(ii) There is an open set $U$ so that $F=\bar{U}$,

(iii) $U$ and $\gamma U$ are either identical or disjoint.

We recall that a fundamental domain for the action of $\Gamma$ on $\mathbb{H}$ is given by the set

$$
F=\left\{z \in \mathbb{H}:-\frac{1}{2} \leqslant \operatorname{Rez} \leqslant \frac{1}{2} \text { and }|z| \geqslant 1\right\}
$$

Moreover, two distinct points $z$ and $w$ of $F$ are equivalent under $S L(2, \mathbb{Z})$. 


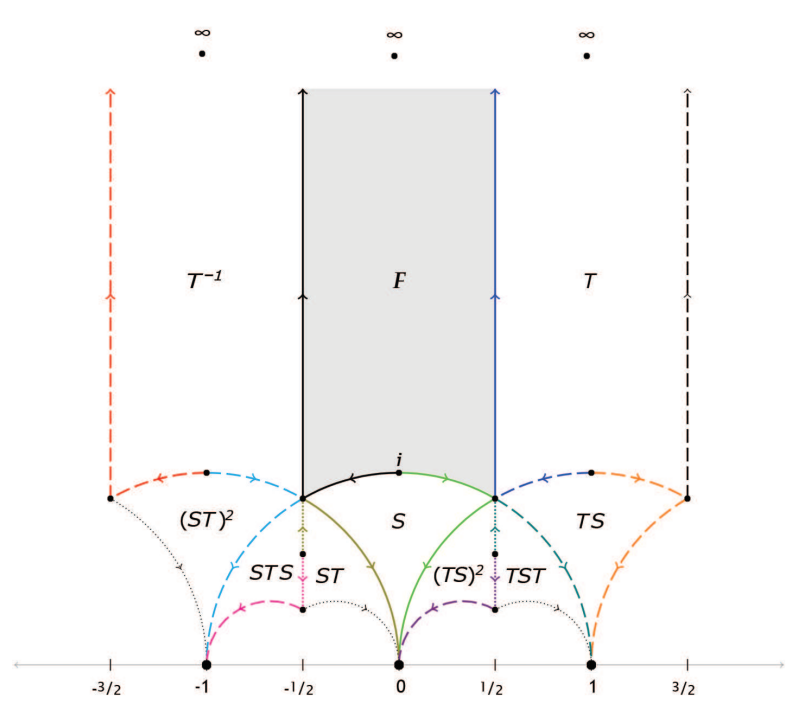

Fig. 1: Fundamental Domain for $\Gamma$.

Let $z \in F$ and let $\lambda(z)=\{\gamma \in \Gamma: \gamma z=z\}$ be the stabilizer of $z$ in $\Gamma$. One has $\lambda(z)=I$, except in the following three cases:

(a) $z=i$, in which case $\lambda(z)$ is the group of order 2 generated by $S$,

(b) $z=w=\exp \left(\frac{2 i \pi}{3}\right)$, in which case $\lambda(z)$ is the group of order 3 generated by $S T$,

(c) $z=-w^{2}=\exp \left(\frac{i \pi}{3}\right)$, in which case $\lambda(z)$ is the group of order 3 generated by $T S$.

The images of $F$ under $\Gamma$ therefore tesselate $\mathbb{H}$, figure 1 shows a picture of $F$ and its images know these tiles. This tiling can be approximated by considering the images of a finite part of the fundamental domain under a finite number of compositions $S \circ T \circ S \ldots$ where each $S$ and $T$ is either a translation by an integer or a reflection. One can easily check that the full modular group acts transitively on $\mathbb{Q} \cup\{\infty\}$; for any $\alpha, \beta$ there exists $T_{0} \in \Gamma$ such that $T_{0}(\alpha)=\beta$. Besides, for a modular group $\Gamma$ we define the set of cusps of $\Gamma$ as the set of $\Gamma$ orbits in $\mathbb{Q} \cup\{\infty\}$. Since $\Gamma$ is of finite index in $S L(2, \mathbb{Z})$ the set of cusps is finite. It turns out that the set of cusps of $\Gamma$ contains exactly the elements missing for the compactification of the quotient $\Gamma \backslash \mathbb{H}$.

Theorem 1. Let $s$ be a cusp. There exists a neighbourhood $U$ of $s$ in $\mathbb{H}^{*}$ with the following property: if $\gamma \in \Gamma$ and $U \cap \gamma(U) \neq \varnothing$, then $\gamma(s)=s$.

Proof. Let $v=\{z \in \mathbb{H}: \operatorname{Im} z>1\} \cup\{\infty\}$. We claim that $U=\alpha(v)$ has the required property. If $U \cap \gamma(U) \neq \varnothing$, then $v \cap\left(\alpha^{-1} \gamma \alpha\right)(U) \neq \varnothing$. But, for all $z \in v \backslash\{\infty\}, \operatorname{Im} z>1$ implies that $\operatorname{Im}\left(\left(\alpha^{-1} \gamma \alpha\right)(z)\right)<1$; thus, $\left(\alpha^{-1} \gamma \alpha\right)(z) \notin v$ and also $\gamma(\alpha(z)) \notin U$. 

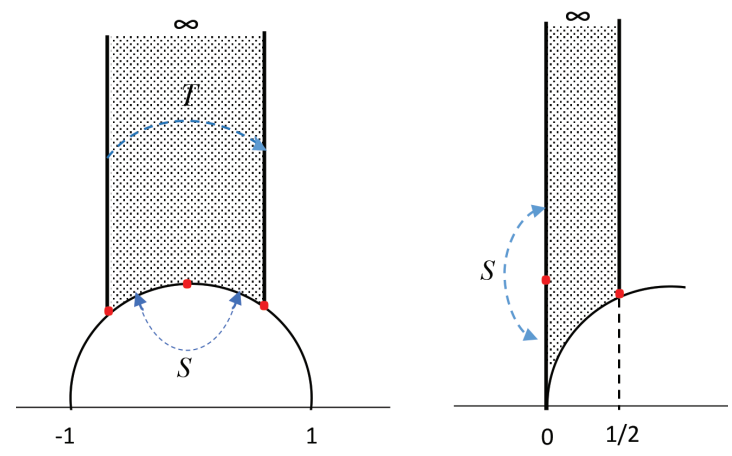

Fig. 2: Paired Edges.

A fundamental domain should be connected.There are several standard choices for a fundamental domain for $S L(2, \mathbb{Z})$ here are two, pictured together with matrices giving identifications of the edges. In the figure elliptic points are marked. And we say that the domain on the right is a better choice for certain theoretical purpose. Fundamental domains are very important in direct integral decompositions for unitary representations. Because one often use particularly fundamental domains as parameters in direct integral decompositions. Therefore for such applications, it is value that a measurable choice be made. Naturally common fundamental domains appear in connection with measure equivalence of groups as well. Hereby, in our aim below of existence of a common fundamental domains is understand.

Lemma 1. Let $G$ be a non discrete subgroup of $\operatorname{PSL}(2, \mathbb{R})$. Then there is no fundamental domain for the action of $G$ on upper half plane.

Proof. Let $t_{n}$ be an arbitrary sequence in $G$ which converges element wise to the identity and also let $F$ be a fundamental domain for the action of $G$. Consider an element $\xi$ of $\operatorname{int}(F)$. Hence $t_{n} \xi$ converges to $\xi$. So there is $m \in \mathbb{N}$ such that $\operatorname{int}(F) \cap t_{m}(\operatorname{int}(F)) \neq \varnothing$, which is impossible.

Theorem 2. Let $\Delta$ be any discrete subgroup of $S L(2, \mathbb{R})$, and assume $D$ be a fundamental domain for $\Delta$. If $\Delta_{0}$ be a subgroup of $\Delta$ of finite index, and choose elements $\delta_{1}, \delta_{2}, \ldots, \delta_{m}$ in $\Delta$ such that $\bar{\Delta}=\bar{\Delta}_{0} \bar{\delta}_{1} \cup \ldots \cup \bar{\Delta}_{0} \bar{\delta}_{m}$, where a bar denotes the image in $A u t(\mathbb{H})$. Then $\bigcup \gamma_{i} D$, for $1 \leq i \leq m$, is a fundamental domain for $\Delta_{0}$.

Proof. Let $z \in \mathbb{H}$. Then $z=\delta w$ for some $w \in \bar{D}, \delta \in \Delta$ and $\delta= \pm \delta_{0} \delta_{i}$ for some $\delta_{0} \in \Delta_{0}$. Thus $z=\delta_{0} \delta_{i} w \in \Delta_{0}\left(\delta_{i} \bar{D}\right)$ where $1 \leq i \leq m$. If $\delta\left(\bigcup \gamma_{i} D\right) \bigcap\left(\bigcup \gamma_{i} D\right) \neq \varnothing$, then it would include a transform of $D$. But then $\delta \delta_{i} D=\delta_{j} D$ for $i \neq j$, which would imply that $\delta \delta_{i}= \pm \delta_{j}$ is obtained. Consequently this is a contradiction.

Corollary 1. The canonical map $\varphi: F \rightarrow \mathbb{H} / \Gamma$ is surjective, and its restriction to int $F$ is injective. In particular, $F$ is a fundamental domain for the action of $\Gamma$ on $\mathbb{H}$.

$\Gamma$ is a Fuchsian group whose fundamental domain has finite area, hence it is known that it has a signature consisting of the geometric invariants $\left(g ; m_{1}, \ldots, m_{r}, s\right)$ where $g$ is the genus of the compactified quotient space, $m_{1}, \ldots, m_{r}$ are the periods of the elliptic elements and $s$ is the parabolic class number. $\Gamma$ has the signature $(0 ; 2,3, \infty)$. The signature of a discrete Fuchsian group is a very interesting problem in group theory and arithmetic-algebraic geometry.

The quotient space of a modular group is known a modular curve and it has the structure of a compact Riemann surface. We denote by $\chi(1)$ the modular curve obtained by the action of $\Gamma(1)$. It is a curve of genus 0 and is rationally equivalent to the projective line. The curve $\chi_{0}(N)$ obtained by the group action of $\Gamma_{0}(N)$ is referred to as the classical modular curve. This case it is important in the theory of elliptic curves because of the theorem of modularity which says that all 
elliptic curves are rational images of classical modular curves. Smallest $N$ such that an elliptic curve is the image of $\chi_{0}(N)$ is called the conductor of the curve. The curve $\chi(1)$ parametrizes isomorphism classes of elliptic curves, while the curve $\chi_{0}(N)$ parametrizes isomorphism classes of elliptic curves with subgroups of order $N$. The genus $g$ of the modular curve $\Omega_{\Gamma}$ can be calculated from the formula:

$$
g=1+\frac{\mu}{12}-\frac{v_{2}}{4}-\frac{v_{3}}{3}-\frac{v_{\infty}}{2}
$$

where $\mu$ is the index of the subgroup $\Gamma(1), v_{2}$ and $v_{3}$ are numbers of elliptic points of order 2 and 3 in $\Gamma$ and $v_{\infty}$ is the number of cusps of $\Gamma$.

Now we take two different congruence subgroups: Firstly, the congruence subgroup

$$
\Gamma_{H}(N)=\left\{\left(\begin{array}{ll}
a & b \\
c & d
\end{array}\right) \in S L(2, \mathbb{Z}): c \equiv 0(\bmod N) \text { and } d \in H\right\}
$$

where $H$ is some subgroup of $\mathbb{Z}_{n}^{*}=\left\{\sigma: \sigma \in \mathbb{Z}_{n},(\sigma, n)=1\right\}$. Obviously if $H=0$ then the group returns $\Gamma_{0}(N)$ and if $H=1$ then the group returns $\Gamma_{1}(N)$. For example we examine the group $\Gamma_{2}(7)$. Generators of this group are as follows:

$$
\left(\begin{array}{ll}
1 & 1 \\
0 & 1
\end{array}\right),\left(\begin{array}{ll}
2 & -1 \\
7 & -3
\end{array}\right),\left(\begin{array}{cc}
4 & -3 \\
7 & 5
\end{array}\right)
$$

and cusps are $0, \infty$. And also genus of $\Gamma_{2}(7)$ is 0 . The index of $\Gamma_{2}(7)$ is finite in $\Gamma$ and $\left|\Gamma: \Gamma_{H}(N)\right|=N \prod_{p \mid N}\left(1+\frac{1}{p}\right)$. Therefore $\left|\Gamma: \Gamma_{2}(7)\right|=8$ is found. We have that $\Gamma=\Gamma_{2}(7) M_{1} \cup \ldots \cup \Gamma_{2}(7) M_{8}$, for a list of right coset representatives $M_{i} \in \Gamma, 1 \leq i \leq 8$.

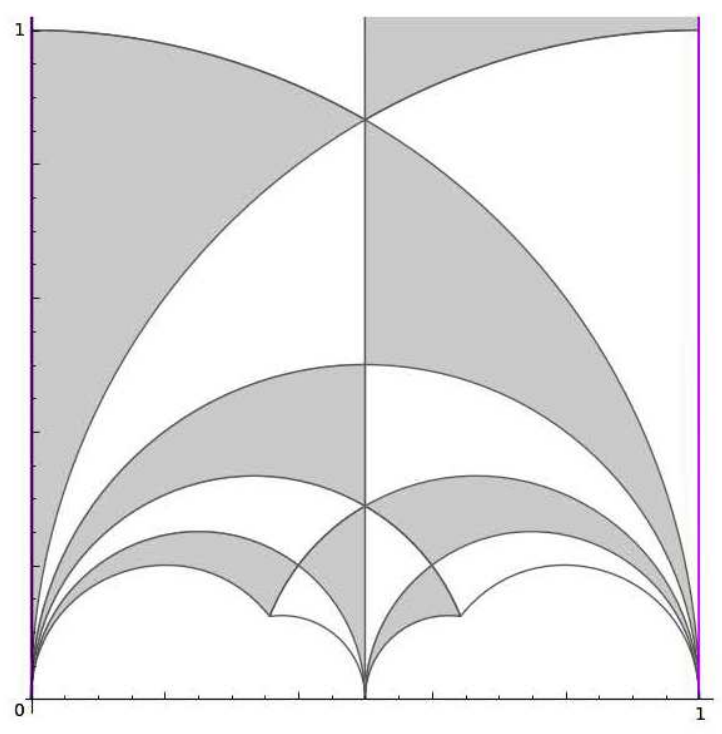

Fig. 3: Fundamental Domain for $\Gamma_{2}(7)$. 
In here we put fundamental domain for $\Gamma_{2}(7)$. This figure is drawn with sage program. Fundamental domains can help us better understand the topological spaces that are the fundamental domains' quotient spaces. In Euclidian space, for instance, the interior of the unit square is a fundamental domain, where the isometries are translation by one unit in the two dimensions.Through their isometries, these fundamental domains tessellate over $\mathbb{H}$. These fundamental domains are not unique. For instance, creating a small indentation on one side of the square and translating the missing piece to the opposite side creates another fundamental domain which behaves exactly the same way.

In addition that the special subgroup of $\Gamma$

$$
\Gamma_{\theta}=\left\{\left(\begin{array}{ll}
a & b \\
c & d
\end{array}\right) \in S L(2, \mathbb{Z}):\left(\begin{array}{ll}
a & b \\
c & d
\end{array}\right) \equiv\left(\begin{array}{ll}
1 & 0 \\
0 & 1
\end{array}\right)(\bmod 2) \text { or }\left(\begin{array}{ll}
a & b \\
c & d
\end{array}\right) \equiv\left(\begin{array}{ll}
0 & 1 \\
1 & 0
\end{array}\right)(\bmod 2)\right\}=\Gamma(2) \cup\left(\begin{array}{cc}
0 & -1 \\
1 & 0
\end{array}\right) \Gamma(2)
$$

is generated by $z \rightarrow \frac{-1}{z}$ and $z \rightarrow z+2$. The subgroup $\Gamma_{\theta}$ is very significant because it appears mostly in sums of squares problems, the simplest application of theta series to seemingly elementary algebraic number theory problems.

Lemma 2. $\Gamma_{\theta}$ has three coset representatives in $\Gamma:\left(\begin{array}{ll}1 & 0 \\ 0 & 1\end{array}\right),\left(\begin{array}{ll}1 & 1 \\ 0 & 1\end{array}\right),\left(\begin{array}{ll}1 & 0 \\ 1 & 1\end{array}\right)$.

Proof. The index is 3 , since $\Gamma_{\theta}$ is index 2 above $\Gamma$ (2). Thus the three listed matrices are in $S L(2, \mathbb{Z}$ and are not only distinct $\bmod 2$ but also do not differ $\bmod \Gamma(2)$ only by multiplication by $\left(\begin{array}{cc}0 & -1 \\ 1 & 0\end{array}\right)$.

Corollary 2. A fundamental domain for $\Gamma_{\theta}$ is $F_{\theta}=\{z \in \mathbb{H}:|\operatorname{Re} z| \leq 1$ and $|z| \geqslant 1\}$.

With usual fundamental domain $F$ for $\Gamma$ the coset representatives for $\Gamma_{\theta}$ in $\Gamma$ give a fundamental domain

$$
F_{\theta}=\left(\begin{array}{ll}
1 & 0 \\
0 & 1
\end{array}\right) F \cup\left(\begin{array}{ll}
1 & 1 \\
0 & 1
\end{array}\right) F \cup\left(\begin{array}{ll}
1 & 0 \\
1 & 1
\end{array}\right) F
$$

We may take more easily describable this form.

Now we give two definitions.

Definition 5. A modular function for $\Gamma$ is a function $f: \mathbb{H}^{*} \longrightarrow \mathbb{P}^{1}$ satisfying the following conditions:

(i) $f$ is invariant under the action of $\Gamma$ on $\mathbb{H}^{*}$, that is, $f \circ \gamma=f$ for all $\gamma \in \Gamma$

(ii) $f$ is meromorphic in $\mathbb{H}$,

(iii) $f$ is meromorphic at the cusps.

Example 1. The index $|\Gamma: \Gamma(2)|=6$. It is possible to find a set of generators for $\Gamma(2)$ just as we found a set of generators for $\Gamma$, and again it suffices to check condition (i) for the generators. There are three inequivalent cusps, namely, $i \infty$, $S(i \infty)=0$ and $T S(i \infty)=1$.The stabilizer of $i \infty$ in $\Gamma(2)$ is generated by $z \rightarrow z+2$, and so $f(z)=f^{*}(\exp (i \pi z))$ for $f$ to be meromorphic at $i \infty$ means $f^{*}$ is meromorphic at 0 . For $f$ to be meromorphic at 0 means that $f(S z)=f\left(\frac{-1}{z}\right)$ is meromorphic at $i \infty$, and for $f$ to be meromorphic at 1 means that $f\left(1-\frac{1}{z}\right)$ is meromorphic at $i \infty$.

Definition 6. A modular form for $\Gamma$ of weight $k$ is a function $f: \mathbb{H}^{*} \longrightarrow \mathbb{P}^{1}$ satisfying the following conditions:

(i) $f$ is weakly modular for $\Gamma$ of weight $k$

(ii) $f$ is holomorphic in $\mathbb{H}$ 
(iii) $f$ is holomorphc at the cusps.

Example 2. The theta series

$$
\theta_{2 k}(z)=\sum_{m_{1}, \ldots, m_{2 k}} e^{i \pi\left(m_{1}^{2}, \ldots, m_{2 k}^{2}\right) z}
$$

are modular forms for $\Gamma_{\theta}$.

We will give only the statement theorem in below.

Theorem 3. Let $f$ be a meromorphic function on $\mathbb{H}^{*}$. The following properties are equivalent:

(i) $f$ is a modular function for $\operatorname{SL}(2, \mathbb{Z})$,

(ii) $f$ is a quotient of two modular forms for $S L(2, \mathbb{Z})$ of the same weight.

Despite the fact that we motivate the definition of modular forms as a means to obtain modular functions, modular forms are interesting on their own right and have many interesting applications in number theory and several other areas of mathematics. For instance Diophantine equations, Eisenstein Series, Fermat's last theorem, construction of Ramanujan graphs, cryptography and coding theory, lattices etc.

Lemma 3. Let $\Gamma$ be a modular group.

(i) If $\eta_{1}$ and $\eta_{2}$ are modular functions then $\eta_{1} \eta_{2}$ is a modular function for $\Gamma$.

(ii) If $\eta_{1}$ and $\eta_{2}$ are modular forms then $\eta_{1} \eta_{2}$ is a modular form for $\Gamma$.

Proof. (i) Let $\eta_{1}$ and $\eta_{2}$ are modular functions. Then, the product $\eta_{1} \eta_{2}$ of two meromorphic functions on the upper half plane is itself meromorphic. That is a weakly modular function. Let $\sum_{n=m}^{\infty} a_{n} q^{n}$ and $\sum_{n=k}^{\infty} b_{n} q^{n}$ be their expansions around any $\alpha \in \mathbb{R} \cup\{\infty\}$, then their formal product is the expansion of $\eta_{1} \eta_{2}$. But the formal product of two Laurent series with convergence in the intersection of the convergent domains of the original series, so $\eta_{1} \eta_{2}$ has a meromorphic expansion at each $\alpha \in \mathbb{R} \cup\{\infty\}$ and hence at each cusp.

(ii) We are in exactly the same case in part (i), but because $\eta_{1}$ and $\eta_{2}$ are modular functions $m, k \geq 0$ and hence the function is holomorphic at each of its cusps.

\section{Competing interests}

The authors declare that they have no competing interests.

\section{Authors' contributions}

All authors have contributed to all parts of the article. All authors read and approved the final manuscript.

\section{References}

[1] M. Akbaş, D. Singerman, Symmetries of modular surfaces, London Mathematical Society Lecture Note Series 173, (1992), 1-9.

[2] M. Beşenk, Connectedness of suborbital graphs for a special subgroup of the modular group, Mathematical Sciences and Applications E-Notes 4,(2016), 45-54.

[3] M. Beşenk, Suborbital graphs for the invariance group, Beykent Uni. Journal of Science and Engineering 10, (2017), 15-29. 
[4] Y. Kesicioğlu, M. Akbaş, M. Beşenk, Connectedness of a suborbital graph for congruence subgroups, Journal of Inequalities and Applications 1, (2013), 117-124.

[5] B.Ö. Güler, T. Köroğlu, Z. Şanl1, Solutions to some congruence equations via suborbital graphs, SpringerPlus 5, (2016), 1-11.

[6] S. Katok, Fuchsian groups, The University of Chicago Press, Chicago and London, 1992.

[7] G.A. Jones, D. Singerman, Complex functions: an algebraic and geometric viewpoint, Cambridge University Press, 1987.

[8] B. Schoeneberg, Elliptic modular functions, Springer, Berlin, 1974.

[9] G. Shimura, Introduction to the arithmetic theory of automorphic functions, Princeton University Press, Princeton, 1971.

[10] W. Stein, Modular forms, a computational approach, American Mathematical Society, Providence, 2007. 